Document downloaded from:

http://hdl.handle.net/10251/146279

This paper must be cited as:

Payri, R.; Giraldo-Valderrama, JS.; Ayyapureddi, S.; Versey, Z. (05-0). Experimental and analytical study on vapor phase and liquid penetration for a high pressure diesel injector. Applied Thermal Engineering. 137:721-728.

https://doi.org/10.1016/j.applthermaleng.2018.03.097

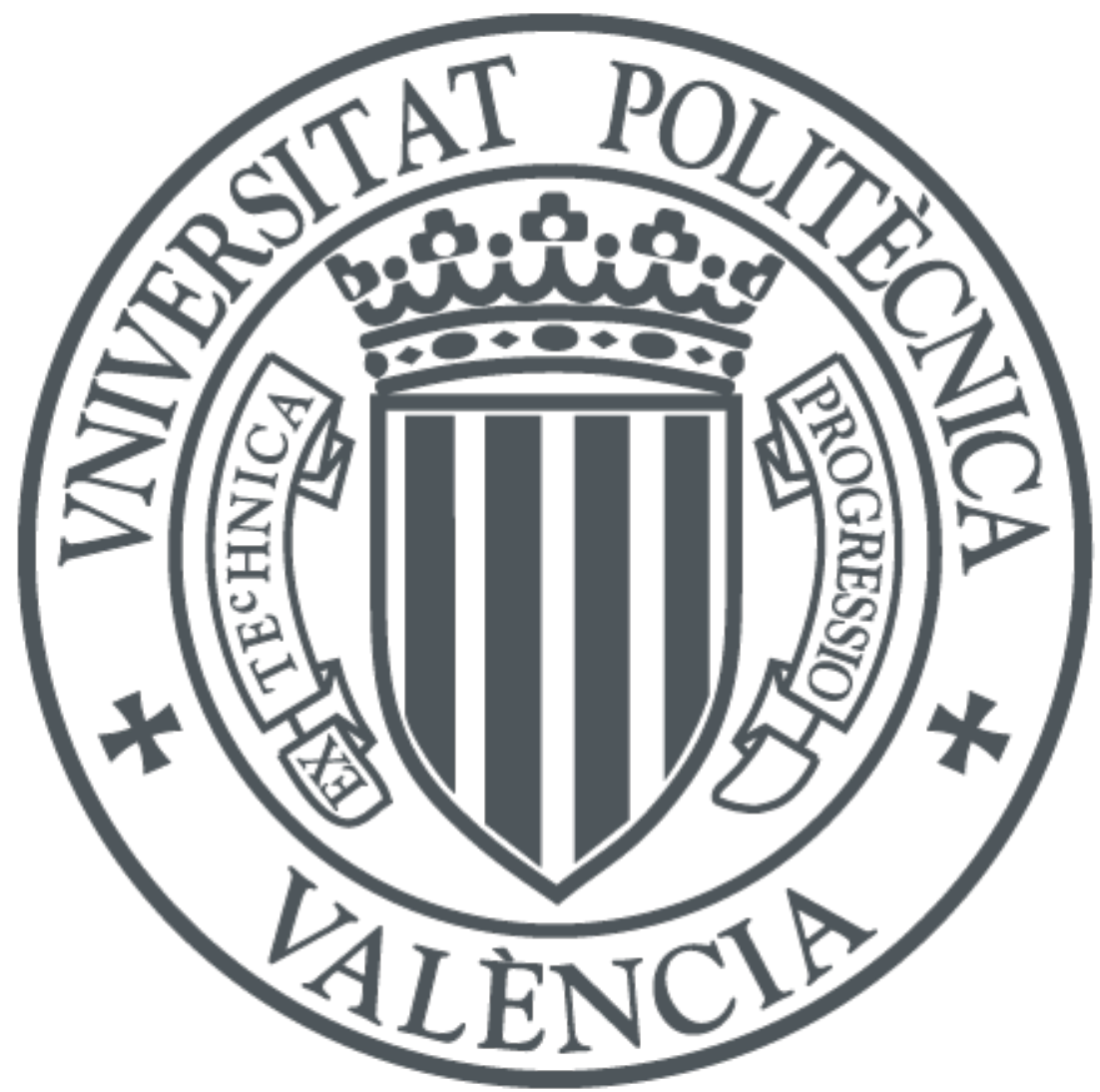

The final publication is available at

https://doi.org/10.1016/j.applthermaleng.2018.03.097

Copyright Elsevier

Additional Information 


\title{
Experimental and analytical study on vapor phase and liquid penetration for a high pressure diesel injector
}

\author{
Raul Payri*1, Jhoan S. Giraldo ${ }^{1}$, S. Ayyapureddi², Z. Versey ${ }^{2}$ \\ 1. CMT - Motores Térmicos, Universitat Politècnica de Valéncia, Edificio 6D, 46022, València, Spain \\ 2. Thermofluids $\&$ Integration, Powetrain, Jaguar Land Rover, Abbey Road, Whitley, Conventry, UK, \\ CV34LF
}

\begin{abstract}
In this study, a macroscopic characterization has been performed on a solenoid diesel injector (2200bar-8 hole nozzle) under various non-reacting but evaporative conditions. For vapor penetration a two pass Schlieren visualization set up was selected. A high speed camera was used to record high speed images of the injection event to analyze the transient evolution of the vapor phase of the spray. The transient liquid penetration of the spray has been measured via MIE-Scattering imaging technique using a high speed camera as well. Unsteady RANS based CFD Simulations have been performed to simulate the experimental conditions and correlation results are presented. Built-in models from commercial code StarCD have been used to model spray formation which includes submodels for turbulence, nozzle flow, break-up and fuel properties. A novel CAE process using an automation and optimization tool has been used to achieve robust model settings, and the final model prediction are compared with the experimental observation for the injector characterization with respect to the non-reacting spray penetration with change in ambient and injection conditions. The model correlates well with the sensitivities for temperature and injection pressures qualitatively however improvements required to capture the density effects mainly related to the mesh orientation, fixed time step size where further analysis required.
\end{abstract}

Keywords: Macroscopic characterization, Diesel spray, Lagrangian two-phase flow, Robust spray model, RANS

* Corresponding author. Tel.: +34 963879658; fax: +34 963877659. E-mail addresses: rpayri@mot.upv.es (R. Payri), jghi@mot.upv.es (Jhoan S. Giraldo),

sayyapu1@jaguarlandrover.com (S. Ayyapureddi), zversey@jaguarlandrover.com (Z. Versey). 


\begin{tabular}{|lc|}
\hline Nomenclature & \\
& \\
RANS & Reynolds Average Navier Stokes \\
$M P I 2$ & Modified Max-Planck-Institute \\
$R N G$ & Renormalization Group \\
$M D O$ & Multi-disciplinary Optimization \\
SHERPA & Simultaneous Hybrid Exploration that is Robust, Progressive and Adaptive \\
$C F D$ & Computational fluid dynamics \\
$R M S E$ & Root mean square error \\
$S O I$ & Start of injection \\
$L / D$ & Nozzle Length-to-Diameter Ratio \\
Mie & Mie-Scattering optical technique \\
\hline
\end{tabular}

\section{Introduction}

Nowadays, internal combustion engines continue to be an important alternative for energy transformation. The ever more demanding fuel consumption standards and the concerns about the environmental impacts of these engines have pushed the industry into the search of new strategies and technologies. This encourages new studies for improving engine performance and its emissions.

The injection process has been mentioned as an important player in order to improve emissions and engine performance. [1 6 . The spray formation includes complex and heterogeneous processes, majorly high-velocity jet flow, liquid droplet break-up, atomization, and evaporation of a dense liquid spray in a turbulent flow environment. The small temporal and spatial scales resulted from this process makes the diesel spray evolution a complicated problem.

To ensure a good mixture between the air and the fuel, the spray must penetrate into the combustion chamber and atomize. There are several parameters that help to characterize the diesel spray from a macroscopic point of view. The liquid length is an indicator of the evaporation capacity of the fuel and it is defined as the distance from the nozzle to the point where are found the ambient conditions necessary for evaporation. Mie scattering imaging technique is widely used by the engine community for the visualization of the fuel spray liquid phase. This technique consists in illuminating the fuel droplets with a light source and collecting the light scattered with a camera [7-9]. The vapor penetration largely determines both the mixing process and the probability of collision against the chamber walls. It depends essentially on the instantaneous momentum of the spray in the nozzle. The Schlieren technique is able to distinguish gradients in the reflective index of a transparent medium [10, 11], which allows clear identification of the vapor phase of the spray in evaporative conditions.

Since diesel combustion is predominantly a mixing-controlled reaction process, modeling the diesel spray formation process accurately is an essential prerequisite for modeling combustion events. The processes involved in the injection event are nonlinear and controlled 
by multiphase, diffusion phenomena. Modeling the interaction between those complex phenomena poses a huge challenge, and obtaining a unique model which can be robust for a wide range of in-cylinder conditions during fuel injection event is important. For industrial application, it is important that the model is viable with computational time and cost. A wide range of numerical models and sub-models exist in the literature [12 19] by various research groups which are inherently different in many aspects. The database is huge and detailed however still limited to the single-hole injector with moderate injection pressures.

In this work, a diesel multi-hole common rail injector (2200 bar) has been modeled using Lagrangian two-phase flow spray model. Relevant turbulence, nozzle flow, break-up models have been selected. Since the properties of diesel used in tests are unavailable various surrogate fuel properties have been applied. The sensitivities of model settings are included in the study, observations are discussed in section 4. A novel CAE process using an automation and optimization tool has been presented which was used to investigate a range of model setting combinations to achieve robust spray model settings. The results from simulations obtained with the optimized parameters, are compared with results from visualization and characterization experiments carried out in this study.

\section{Model development}

\subsection{CFD Methodology}

The numerical simulations are performed using the commercial CFD tool Star-CD. The turbulent flow field is resolved using the $\mathrm{k}-\epsilon$ equation based Renormalization Group (RNG) turbulence model as this is in-cylinder combustion best practice [20]. The Lagrangian based two-phase flow model has been used to resolve spray formation. The nozzle inflow models are used to capture the nozzle hole exit velocities, the two models considered here are the Effective and MPI2 (modified Max-Planck-Institute) [21, 20] models from Star-CD. The advantage of the MPI2 model is that it automatically determines whether cavitation occurs inside the nozzle and distinguishes whether it reaches the nozzle exit or ends inside the nozzle. For all simulations in this paper, the properties of n-dodecane $\left(\mathrm{C}_{12} \mathrm{H}_{26}\right)$ were used as a surrogate for diesel fuel, these were taken from the internal StarCD fuels library [22]. The properties of the surrogate can be seen in table 1 . 
Table 1: n-Dodecane properties @ 298.15 K \& 101325 Pa

\begin{tabular}{lcc}
\hline Properties & Value & Units \\
\hline Molecular weight & 170 & $\mathrm{~kg} / \mathrm{mol}$ \\
Critical temperature & 658.65 & $\mathrm{~K}$ \\
Critical pressure & $1.835 \times 10^{6}$ & $\mathrm{~Pa}$ \\
Boiling temperature & 489.48 & $\mathrm{~K}$ \\
Density & 745.76 & $\mathrm{~kg} / \mathrm{m}^{3}$ \\
Molecular viscosity & 0.00137563 & $\mathrm{~kg} / \mathrm{ms}$ \\
Surface tension coefficient & 0.0248679 & $\mathrm{~N} / \mathrm{m}$ \\
\hline
\end{tabular}

The injected liquid with high velocity starts to break-up into smaller droplets, the process comprises of primary breakup (i.e. atomization) and secondary breakup. A range of built-in sub-models is available with-in Star-CD to model this phenomenon. Atomization models differ in the way droplet size distribution and initial velocities are calculated. The difference between the droplet break-up models is the correlations that are used to estimate the time scale of the break-up process and the stable droplet diameter. The Huh atomization model and Reitz -Diwakar droplet break-up models have been used [20]. The Huh model calculates the spray cone angle during simulation so this is not required as an input.

The heat and mass transfer process is modeled using Ranz-Marshall correlation 23] to capture the evaporation process. The drag process and turbulence dispersion are modeled using stand correlation [20]. The inter-droplet collisions are not modeled, as the RNG turbulence model does not take this into account [20]. The droplet-wall interaction is not significant in this bomb case setup, however, the Bai model has been selected to consider any such process [22].

\subsection{Computation grid and boundary conditions}

The 3D computational domain used to represent the spray chamber fluid volume has been created as shown in Fig.1. The boundary with the injector is defined an adiabatic wall whereas the other boundaries are defined as pressure-outlets. The dimensions of the cuboid are maintained to enclose the non-reacting spray from the multiple injector holes whilst minimizing the influence of the boundary wall on the spray. The location of injector hole is defined at the center of the domain, at a certain depth below the wall surface for the same reason. All the dimensions and characteristics of the computational domain can be found in [22].

The coordinate system seen in Fig 11 represents each of the 8 holes of the injectors, and was used to set the injection locations within the domain based on the geometrical specification of the injector. A uniform grid with cell size $0.8 \mathrm{~mm}$ has been selected as these are the settings used within full combustion models within the JLR 3D-thermofluids 
diesel combustion group, for which the tuned spray model parameters are required [22].

\subsection{Automation and optimization: HEEDS}

In this section, HEEDS is briefly explained. For this study the HEEDS MDO (MultiDisciplinary Optimization) software was utilized in two manners, firstly to carry out a DoE (design of experiment study) into a set of tuning factors to investigate the effects of each on the penetration, and secondly to provide an automated workflow and optimization methodology to target a CFD solution which matches the experimental observations for a specific operating condition. SHERPA is the main algorithm used for optimization; the basic mechanism is that algorithm uses the results from numerical simulations to adapt to a new search path after each run and thus the number of evaluations required to arrive to given target performance can be quite different from run to run. The detailed mechanisms of algorithm is intellectual property of the Red Cedar technologies, however the high level description of the algorithm can be found in 24]. The SHERPA algorithm automatically applies the appropriate optimization algorithms for the problem based on what it has learnt about the design space in the previous results. The design space is navigated as the optimization algorithm performs real CAE analyses, rather than an approximated surrogate model. Without the need to generate a surrogate model, the number of analysis runs required is reduced, saving time and resources. Other advantages of using this method include the fact that the user does not need to understand the design space to select an appropriate algorithm prior to starting to the optimization, nor does the user need to have any expertise in optimization applications.

Within the HEEDS GUI an automatic process is setup, instructing the software which step is the following to execute for the optimization. First, the input parameters to be varied, for the spray characterization, were selected and tagged for editing in Star-CD model example input files. Similarly, the responses are created and tagged in example output files, the penetrations for liquid and vapor are written out to a database file using an user sub-routine. The main responses in this study are liquid penetration and vapor penetration. Experimental data of liquid and vapor penetration is read into HEEDS as target curves $\left(Y_{t}\right)$ and the simulation output of liquid and vapor penetration were tagged as design curves $\left(Y_{d}\right)$. HEEDS using the equation below for the Root Mean Squared Error (RMSE) to find by targeting the minimum value for each response from the entire design space.

$$
R M S E=\sqrt{\frac{1}{n} \sum_{i=1}^{n}\left(Y_{d}+Y_{t}\right)^{2}}
$$

Finally a study is setup in which the variables and responses to be taken into account in the optimization are set, as well as targets (which can be weighted based on importance) and constraints for the solver. For the DoE, the low/high values for each input factor were given and no constraints or objectives were needed. For the calibration run no constraints were set, and a standard SHERPA parameter optimization with weighted curves were 
used, and the objectives were set to minimize the "Liquid" and "Vapor" responses, i.e. to minimize RMSE. The details of variables and their levels for respective studied are presented in detail in table 3 and 5 .

\section{Experimental set up}

\subsection{The high pressure and high temperature test rig and fuel injection system}

The feeding system for the fuel consists of a conventional common rail configuration that contains a high pressure pump and a rail with a pressure regulator. This system allows injections at high pressures up to 2200 bar. The measures were carried out in a test vessel classified as a constant-pressure flow (CPF) facility (fig.2), where the thermodynamic conditions of the engine combustion chamber can be reproduced [25]. The gas is stored by volumetric compressors in high pressure reservoirs and flows continuously through the test chamber. To keep the gas in the test section at the desired temperature, $30 \mathrm{~kW}$ electrical heaters were placed upstream of the chamber. The control system is a closed loop PID that adjusts both the pressure in the chamber and the power of the heaters to obtain the test conditions required for the experiments.

This test rig allows a maximum ambient temperature of $1000 \mathrm{~K}$ and a maximum pressure of 150 bar. The gas at high pressure and temperature continuously flow through the chamber at $0.3 \mathrm{~m} / \mathrm{s}$. The test rig has three large windows $(128 \mathrm{~mm}$ diameter) that give full optical access, and the big chamber diameter $(200 \mathrm{~mm})$ minimizes the spray-wall interaction. In this study, the vessel has been filled with nitrogen to guarantee the evaporative but non-reacting conditions sought.

\subsection{Optical set up for vapor penetration (Schlieren-based)}

The Schlieren imaging technique was used to identify the spray vapor phase boundaries at evaporative conditions. The technique is based on the change of refraction of parallel light rays that pass through non-homogeneus fluids checking density variations 26]. The refractive index gradient into the region of interest will cause the deviation of some rays. Using a spherical lens to collect the beam, only parallel rays will converge to the focus point of the lens. Then parallel rays can be identified and trimmed using a diaphragm at the focus point, obtaining a shadowgraphic image. For this test a double-pass Schlieren configuration using a high temperature mirror has been used. The set up for this configuration can be seen in fig.2. The main difference of the optical arrangement for a double-pass Schlieren setup, is the fact that the rays are passing two times through the test section, being reflected by the mirror placed right behind the test section. Since the light is reflected by the mirror toward the same direction it is coming from, a beam-splitter is required to complete the setup and reflect the image to the camera [26, 27, 11] 


\subsection{Optical set up for liquid penetration (Mie-scattering-based)}

The MIE scattering optic technique was used to identify the spray liquid phase boundaries. It consists in illuminating the spray with a light source (continuous or pulsed) so the scattered light could be collected by a fast camera.

In this work, the sprays have been illuminated by the front window with two continuous Xe-arc lamps and the light scattered backward was collected by a high speed CMOS camera (Phantom v12) aligned with the injector axis. The size of the images were 768 $\mathrm{x} 712$ pixel with a spatial resolution of $5.41 \mathrm{pixel} / \mathrm{mm}$. The acquisition rate was $24 \mathrm{kfps}$ The set up for this configuration can be seen in fig. 3 .

\subsection{Image Processing}

Each image recorded is first divided into eight sectors, one for each outlet orifice and thus one spray. In this way, each spray is processed separately by applying mask to isolate the spray of interest. The algorithm used for the processing is described in [11, 28]. The image is inverted in order to have the spray as the high luminosity area and the threshold is calculated as the $3 \%$ of that image's dynamic range [11, 29. Consequently, small areas that come from background noise are ruled out and finally the spray contour is "cleaned" free of small noise fluctuations through a pixel connectivity evaluation. This last step could be seen as a contour smoothing. Liquid penetration (Mie scattering) and vapor penetration (Schlieren) are calculated by detecting the pixel on the contour that is the furthest from the outlet orifice; the penetration is then calculated as the axial distance from the injector outlet to the furthest point [27].

\subsubsection{For Mie-Scattering}

The steps followed in the image processing for the Mie-scattering study are summarized below [11]:

1. The image acquired right before the start of injection is arithmetically subtracted from the spray images, in order to remove reflections and background artifacts.

2. In order to analyze each spray individually, the image has been divided in 8 sectors.

3. The contour of each spray is obtained using a variable threshold (ths). The threshold is calculated as the $3 \%$ of the dynamic range of the sector.

4. Applying the threshold the image is binarized. The connectivity algorithms are employed to distinguish between the spray and the artifacts due to sensor noise [11, 28.

5. The spray boundary is finally obtained as the contour of this area.

\subsubsection{For Schlieren}

For the image segmentation, it was used the same approach followed in Mie scattering tests: the image was separated in sectors to process each spray separately, the background subtraction was applied and a black and white image was obtained using a scaled threshold 
as in Mie scattering. However, the images obtained in Schlieren are characterized by features that required to modify the processing routine used for the Mie images. Some considerations about this kind of tests are [11]:

1. The spray appears darker than the background, therefore an inversion of the image and of the background is convenient.

2. The head of the three bolts holding the mirror appears as dark spots in the image. Therefore, the sprays interfering with this bolts will not be processed to avoid erroneous measurements.

3. The temperature/density gradient related to the turbulent flow appears in the background and caused fluctuations over a wide range of counts level. Connectivity algorithms have been modified to obtain accurate spray boundaries.

\subsection{Test Plan}

A wide range of conditions have been explored in order to study the spray penetration and are summarized in table 2 ,

Table 2: Experimental test program.

\begin{tabular}{lcc}
\hline Parameter & Value-type & Units \\
\hline Fuel & Diesel & - \\
Energizing time & 1500 & $\mu \mathrm{s}$ \\
Intercoolant temp. & 363 & $\mathrm{~K}$ \\
Ambient gas density & $20,25,30$ & $\mathrm{~kg} / \mathrm{m}^{3}$ \\
Ambient gas temp. & $600,800,900$ & $\mathrm{~K}$ \\
Injection pressure & 1100,1500, & $\mathrm{bar}$ \\
& 1800,2200 & \\
Oxygen concentration & 0 & $\%$ \\
\hline
\end{tabular}

\section{Results}

\subsection{DoE Investigation}

The DoE investigation results are presented in this section. For this study, it was decided that a 2 level full factorial investigation would be carried out, meaning that 256 designs needed investigating, a task which could not have been done by hand, due to the time it would have taken to set up and post process each simulation. The factors included 2 nozzle flow models, Reitz-Diwakar break-up model factors Te-Strip (refers to the empirical coefficient Cs2 of characteristic time scale of stripping break-up regime [20, 30] and WeBag (refers to the empirical coefficient Cb1 to determine the stable droplet size for Bag 
break-up [20, 30], along with other injector nozzle parameters with potential geometrical uncertainties. For the DoE, the low and high values for the investigation were set in HEEDS as shown in table 3 ,

Table 3: Range of parameters used in HEEDS DoE Investigation

\begin{tabular}{lccc}
\hline Factor & Model parameter & Low & High \\
\hline $\mathrm{A}$ & $\mathrm{L} / \mathrm{D}$ & 4 & 8 \\
$\mathrm{~B}$ & $\mathrm{Cd}$ & 0.7 & 0.8 \\
$\mathrm{C}$ & Contraction ratio & 0.5 & 0.6 \\
$\mathrm{D}$ & Te-Strip & 9 & 20 \\
$\mathrm{E}$ & Injection Temperature & 330 & 353 \\
$\mathrm{~F}$ & Injection Parcels & $1 e+07$ & $2 e+07$ \\
$\mathrm{G}$ & We-bag & 3.6 & 8.4 \\
$\mathrm{H}$ & Nozzle Model & $1-$ Effective Model & $3-$ MPI2 Model \\
\hline
\end{tabular}

From an interaction point of view the DoE results show us that Te-Strip and nozzle model have the greatest interactions of all the factors for both the liquid and vapor. On the other hand, the number of injection parcels has very little effect on the results nor does it interact with any of the other factors, similar trends were seen with the injection temperature and contraction ratios.

From the main effects plots in Fig $4 \mathrm{a}$ and $4 \mathrm{~b}$, it is clear that the importance of each factor for the liquid and vapor penetrations are not equal. Te-Strip has the largest effect on both, followed by the nozzle model. The nozzle interaction for liquid and vapor is reversed, in other words the user must choose between a better match to experiment for liquid or for vapor but cannot do both at the same time.

In running this DoE, the injector hole diameter was fixed, however, the effective nozzle model requires the diameter to be reduced as it does not take account of cavitation inside the nozzle hole. An additional study was carried out using the 'best' settings from the DoE results with the effective nozzle model whilst sweeping the diameter from geometrical $(0.000144 \mathrm{~m})$ down to minus $10 \%$ of geometrical $(0.00013 \mathrm{~m})$. The results of the sub-study showed that the effective nozzle can provide a better result than the DoE for Vapor RMSE, whilst simultaneously preserving (or improving) the RMSE for Liquid (compared to the DoE when optimized for vapor). In other words, the Effective nozzle sweep provides a better trade-off, maintaining the best results from the DoE for both liquid and vapor. The final best settings taken forward to the CFD comparison to experimental have been listed in the table 4. 
Table 4: "Best" settings taken from study.

\begin{tabular}{lc}
\hline Model parameter & Best setting \\
\hline L/D & 9 \\
Cd & 0.8 \\
Contraction ratio & 0.55 \\
Te-Strip & 20 \\
Injection Temperature & 353 \\
Injection parcels & $1 \mathrm{e}+07$ \\
We-Bag & 8.4 \\
Nozzle model & Effective model \\
Hole diameter & 0.00013 \\
\hline
\end{tabular}

\subsection{Experimental to CFD Comparison}

\subsubsection{Effect of ambient density on vapor and liquid penetration}

It has been reported before that for both liquid and vapor penetrations, ambient density is a crucial parameter $[31-33$. The figures $5 a$ and $5 b$ reflect this behavior and show that an increase in density causes a slower spray penetration. A higher density of the entrained gas requires more kinetic energy to achieve the momentum transfer, and for this reason the spray penetrates slower.

The CFD spray model with the stated best settings captures the trend of density effect on the spray penetration qualitatively, although vapor penetration over-predicts and liquid penetration under-predicts.

Further analysis is required to improve the predictions quantitatively, mainly improving the overshoot in the liquid penetration before steady spray is achieved. It is believed this could be due to the fact the cell size and time-step size chosen is slightly too large to correctly capture the initial spray penetration phase.

\subsubsection{Effect of Injection pressure on vapor and liquid penetration}

As previously exposed in the table 2, two injection pressures were studied during the experimental stage. fig 6 illustrates the influence of the injection pressure. As expected, an increase in injection pressure produces an increase in vapor penetration rates, whilst having relatively little impact on the liquid penetrations.

The CFD model reproduces the injection pressure effects, for vapor the penetration rates are increased for the 2200bar injection, and the liquid steady spray for the 2200bar is slightly lower than for the 1100bar injection. However the CFD over estimates the difference between the steady sprays for both injection pressures.

Once again the liquids steady sprays are under-predicted whilst the vapor spray penetration trends are over-predicted. 


\subsubsection{Effect of ambient temperature on vapor and liquid penetration}

For this study two ambient temperatures were selected (fig.7), and the effects on vapor and liquid length penetrations were verified. Naturally, the ambient temperature does not affect vapor penetration significantly, since the key parameters determining the penetration were kept constant in the comparison (injection pressure and ambient density). However, the figure shows a subtle consistent trend, indicating a slight decrease in vapor spray penetration with increasing temperature. This behavior has been reported before [34, and it is maybe caused by the reduction in droplet size due to the evaporation at higher temperatures, which may facilitates the momentum transfer from the fuel to the surrounding air.

For liquid penetration as expected, curves for each temperature overlap in the first transient part since the density is the same in the two cases. However, the liquid length penetration stabilizes at different values as a result of the evaporation fuel at higher temperatures. The subtle effect on vapor penetration and significant effect on liquid penetration due to change in ambient temperature have been well captured by the CFD model, which suggests the model sensitivity vs. the change in temperature is good enough to be used in diesel-like in-cylinder conditions.

\subsubsection{Effect of density and temperature on maximum liquid length penetration}

In fig 8 two different trends for the maximum liquid penetration can be seen. The first trend describes a decrease of the maximum penetration with increased temperature, as it has been explained in the previous section. The second trend seen in the experimental data is a linear decrease of the maximum penetration with increased density.

The CFD model captures the sensitivity due to change in temperature very well. However, it is less capable when it comes to the sensitivity due to the change in density, initially showing a decrease between $20 \mathrm{~kg} / \mathrm{m}^{3}$ and $25 \mathrm{~kg} / \mathrm{m}^{3}$, but then rising again at $30 \mathrm{~kg} / \mathrm{m}^{3}$. It is thought this could be due to the mesh not being spray oriented, hence causing discrepancies in the spray momentum predictions. Further analysis is needed to understand the spray orientation effects.

\subsection{Model calibration study}

A further study was carried out using the HEEDS optimization features to calibrate a model to a specific operating condition to obtain a new set of tuned settings [22]. To do so the experimental data was offset so that both had SOI $=0$ as this is what the CFD is set to. For this study the number of factors for tuning was reduced, using the results of the DoE to remove those with little effect on the results. In total 50 models were run. The table 5 outlines which factors were used and the ranges set for each, and the best settings found from the calibration. All other factors were either set to best practice or those found in the DoE. The deviation in the initial liquid penetration could pertain to the Eularian - Lagrangian approach, where the initial velocity (estimated using nozzle flow sub-model) and time-step lead to droplet parcels with higher momentum which 
penetrates farther. Velocity profile from detailed nozzle in-flow simulation and variable time-step could improve the results. A further detailed analysis has been carried out to check the model related uncertainties to understand this phenomenon

Table 5: Factors and ranges used for calibration

\begin{tabular}{lccccc}
\hline Parameter & Minimum & Baseline & Maximum & Interval & Best Settings \\
\hline L/D & 4 & 8 & 8 & 0.05 & 4.65 \\
Cd & 0.61 & 0.85 & 0.9 & 0.01 & 0.77 \\
Te-Strip & 2 & 13 & 20 & 0.1 & 19.7 \\
We-bag & 3.6 & 8.4 & 8.4 & 0.1 & 5 \\
Hole Diameter & 0.00013 & 0.000144 & 0.000144 & 0.000001 & 0.000144 \\
\hline
\end{tabular}

A direct comparison of the liquid and vapor penetrations between experimental, DoE tune, CFD results, and the calibrated model settings CFD results has been plotted in fig.9. We can see that the calibrated settings give a better steady state liquid penetration match than the DoE settings, however the initial rise in penetration is somewhat delayed. For vapor, the difference is less extreme, though the calibrated model does appear to better match the shape of the experimental curve than the DoE settings. It was observed that the SOI for the experimental data may have been moved too far during the pre-processing. The measured SOI of the experimental data is extrapolated from the measured data and so there will be a margin of error. The largest difference in factor settings between the DoE best settings and the HEEDS calibration settings are for L/D (8 to 4.65) and WeBag (8.4 to 5). The injector hole diameter is also different. The HEEDS optimization has selected the geometrical value, whereas we would normally expect to have to decrease this to account for cavitation.

Overall the main differences in the two settings have a greater effect on the liquid penetration than vapor penetration. The increase in hole diameter for the calibrated from DoE settings results in a reduction of droplet velocities at nozzle exit and hence lower break-up and evaporation, hence deeper penetrations. Further work is required to understand how further optimization should be run in terms of numbers of designs requested, weighting of targets, and looking into the experimental uncertainties related to vapor and liquid measurements.

\section{Conclusions}

A solenoid 8-hole 2200 bar diesel injector has been characterized from a macroscopic point of view by means of Mie-Scattering and Schlieren optical techniques. The effects of ambient temperature, injection pressure and ambient density were studied in a constant flow high pressure and high temperature test rig. The facility emulates in-chamber conditions at the time of injection by means of pressurized and heated gas, to a maximum pressure 
and temperature of 150 bar and $1000 \mathrm{~K}$ respectively.

As expected, injection pressure affects the vapor penetration but no so much liquid penetration. A negligible decrease in vapor penetration has been found by increasing temperature, this is maybe caused by the reduction in droplet size at higher temperatures, which facilitates the momentum transfer.Both liquid and vapor penetration decrease with an increase in the ambient density. This is due to higher momentum transfer at higher densities.

On the other hand, 3D CFD simulations were performed using the built-in sub-models from commercial software StarCD. The spray model DoE investigation showed sensitivity and interaction of various factors, where nozzle flow model and break-up model factors have significant effect compared to other parameters. A robust model setting has been used to run different conditions, and the model results were correlated with experimental data.

In summary, the CFD model is capable of capturing trends in the penetrations as per the experimental data regardless of whether or not the model have been calibrated to those conditions. Overall, the steady state liquid penetrations are under predicted by the model and vapour over-predicts. This is because HEEDS exploration obtains a common model setting that fits best for both liquid and vapor penetration. Extending further this exploration study might results in obtaining combination of breakup model parameter might achieve better match with experiment results. It has also been noted during the work carried out, that the vapour penetration is less sensitive to changes in the factors investigated than the liquid length. The model reproduces the key effects that are observed in experimentation, such as injection pressure dominance on the vapour length and temperature influence on the liquid length, however the sensitivity with density change is marginal captured. Further model calibration and understanding the mesh orientation effects is needed to improve the predictability. To conclude, the CFD results suggest that when time or data is not available to calibrate the model, the DoE settings could be used as a baseline as they appear fairly robust.

\section{Acknowledgments}

This research has been partially funded by FEDER and Spanish Ministerio de Economía y Competitividad through project TRA2015-67679-c2-1-R. Additionally Jhoan Sebastián Giraldo had a grant FPI-SUB 2 from Universitat Politècnica de València.

\section{Bibliography}

[1] Joonsik Hwang, Youngsoo Park, Kihyun Kim, Jinwoo Lee, and Choongsik Bae. Improvement of diesel combustion with multiple injections at cold condition in a constant volume combustion chamber. Fuel, 197:528 - 540, 2017.

[2] Wenbin Yu, Wenming Yang, and Feiyang Zhao. Investigation of internal nozzle flow, 
spray and combustion characteristics fueled with diesel, gasoline and wide distillation fuel (WDF) based on a piezoelectric injector and a direct injection compression ignition engine. Applied Thermal Engineering, 114:905 - 920, 2017.

[3] Nicholas Neal and David Rothamer. Measurement and characterization of fully transient diesel fuel jet processes in an optical engine with production injectors. Experiments in Fluids, 57(10):155, 2016.

[4] Raul Payri, Juan Pablo Viera, Venkatesh Gopalakrishnan, and Patrick G. Szymkowicz. The effect of nozzle geometry over ignition delay and flame lift-off of reacting direct-injection sprays for three different fuels. Fuel, 199:76-90, 2017.

[5] S.N. Soid and Z.A. Zainal. Spray and combustion characterization for internal combustion engines using optical measuring techniques: A review. Energy, 36(2):724741, 2011.

[6] Xiwen Wu, Jun Deng, Huifeng Cui, Fuying Xue, Liying Zhou, and Fuqiang Luo. Numerical simulation of injection rate of each nozzle hole of multi-hole diesel injector. Applied Thermal Engineering, 108:793 - 797, 2016.

[7] Raul Payri, Jose M. García-Oliver, Michele Bardi, and Julien Manin. Fuel temperature influence on diesel sprays in inert and reacting conditions. Applied Thermal Engineering, 35:185 - 195, 2012.

[8] F. Payri, R. Payri, M. Bardi, and M. Carreres. Engine combustion network: Influence of the gas properties on the spray penetration and spreading angle. Experimental Thermal and Fluid Science, 53:236 - 243, 2014.

[9] Raul Payri, Jaime Gimeno, Michele Bardi, and Alejandro H. Plazas. Study liquid length penetration results obtained with a direct acting piezo electric injector. Applied Energy, 106(0):152 - 162, 2013.

[10] G. S. Settles. Schlieren and shadowgraph techniques: visualizing phenomena in transparent media. Springer Verlag, 2001.

[11] Raul Payri, Jaime Gimeno, Juan P. Viera, and Alejandro H. Plazas. Needle lift profile influence on the vapor phase penetration for a prototype diesel direct acting piezoelectric injector. Fuel, 113(0):257 - 265, 2013.

[12] X. Jiang, G.A. Siamas, K. Jagus, and T.G. Karayiannis. Physical modelling and advanced simulations of gas-liquid two-phase jet flows in atomization and sprays. Progress in Energy and Combustion Science, 36(2):131 - 167, 2010.

[13] Yuanjiang Pei, Evatt R. Hawkes, and Sanghoon Kook. Transported probability density function modelling of the vapour phase of an n-heptane jet at diesel engine conditions. Proceedings of the Combustion Institute, 34(2):3039 - 3047, 2013. 
[14] Rickard Solsjö and Xue-Song Bai. Injection of fuel at high pressure conditions: Les study. In SAE Technical Paper. SAE International, 092011.

[15] Raul Payri, Juan Pablo Viera, Yuanjiang Pei, and Sibendu Som. Experimental and numerical study of lift-off length and ignition delay of a two-component diesel surrogate. Fuel, 158:957-967, 2015.

[16] S. Som and S.K. Aggarwal. Effects of primary breakup modeling on spray and combustion characteristics of compression ignition engines. Combustion and Flame, 157(6):1179 - 1193, 2010.

[17] Yue Wang, Hai-Wen Ge, and Rolf D. Reitz. Validation of mesh- and timestepindependent spray models for multi-dimensional engine cfd simulation. SAE Int. J. Fuels Lubr., 3:277-302, 042010.

[18] Haobu Gao, Xiangrong Li, Jiye Xue, Honglin Bai, Xu He, and Fushui Liu. A modification to the $\{\mathrm{WAVE}\}$ breakup model for evaporating diesel spray. Applied Thermal Engineering, 108:555 - 566, 2016.

[19] Kun Lin Tay, Wenming Yang, Feiyang Zhao, Wenbin Yu, and Balaji Mohan. Effects of triangular and ramp injection rate-shapes on the performance and emissions of a kerosene-diesel fueled direct injection compression ignition engine: A numerical study. Applied Thermal Engineering, 110:1401 - 1410, 2017.

[20] MDX. STAR-CD Version 4.08 Documentation. MDX, 2008.

[21] F Obermeier. Modeling of nozzle-flow. IDEA project, subprogram A, 1:1991, 1991.

[22] S. Ayyapureddi, Z. Versey, P. Dunkley, and S.R. Pierson. Development of robust diesel spray model using spray characteri-zation experiment data. THIESEL, 2016.

[23] W. Ranz and W. Marshall. Evaporation from drops: parts i \& ii. Chemical Engineering Progress, 1952.

[24] http://www.redcedartech.com/pdfs/SHERPA.pdf.

[25] Raul Payri, Francisco Javier Salvador, Jaime Gimeno, and Jesús E Peraza. Experimental study of the injection conditions influence over n-dodecane and diesel sprays with two ECN single-hole nozzles. Part II: Reactive atmosphere. Energy Conversion and Management, 126:1157-1167, 2016.

[26] Raul Payri, Juan Pablo Viera, Venkatesh Gopalakrishnan, and Patrick G. Szymkowicz. The effect of nozzle geometry over the evaporative spray formation for three different fuels. Fuel, 188:645-660, 2017.

[27] R. Payri, J. Gimeno, G. Bracho, and D. Vaquerizo. Study of liquid and vapor phase behavior on diesel sprays for heavy duty engine nozzles. Applied Thermal Engineering, 107:365 - 378, 2016. 
[28] V. Macian, R. Payri, A. Garcia, and M. Bardi. Experimental evaluation of the best approach for diesel spray images segmentation. Experimental Techniques, 36(6):2634, 2012.

[29] Payri R., Salvador J., Gimeno J., and Viera A. Effect of injection rate shaping over diesel spray development in non-reacting evaporative conditions. Tschöke H., Marohn R., 2017.

[30] Rolf D. Reitz and R. Diwakar. Effect of drop breakup on fuel sprays. In $S A E$ Technical Paper. SAE International, 021986.

[31] J. Naber and D. Siebers. Effects of gas density and vaporization on penetration and dispersion of diesel sprays. SAE Paper, 1996.

[32] Jose M. Desantes, J. V. Pastor, Raul Payri, and Jose M. Pastor. Experimental characterization of internal nozzle flow and diesel spray behavior. part ii: evaporative conditions. Atomization and Sprays, 15(5):517-544, 2005.

[33] I.V. Roisman, Lucio Araneo, and C. Tropea. Effect of ambient pressure on penetration of a diesel spray. International Journal of Multiphase Flow, 33(8):904 - 920, 2007.

[34] M. Bardi. Partial needle lift and injection rate shape effect on the formation and combustion of the diesel spray. PhD thesis, Universitat Politècnica de València, 2014. 


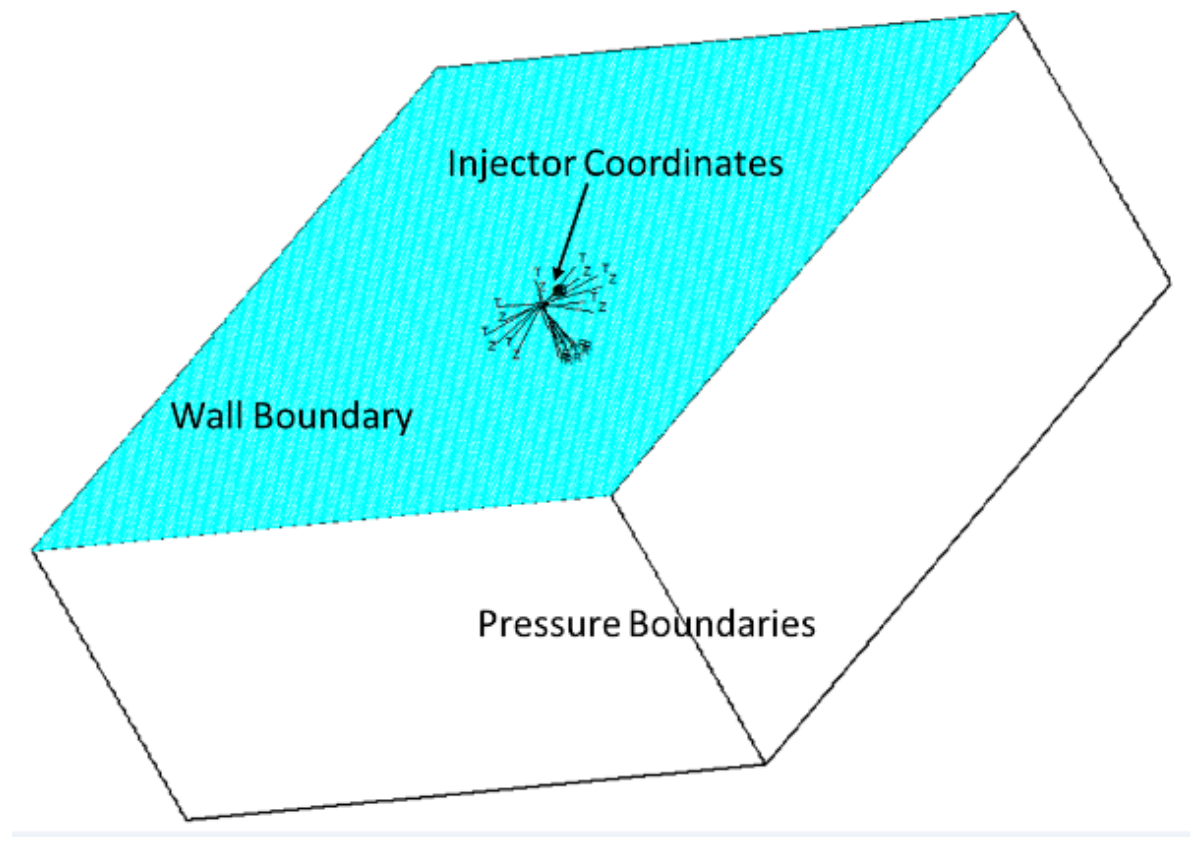

Figure 1: Domain Configuration

Published in Applied Thermal Engineering, Volume 137, 5 June 2018, Pages 721-728 


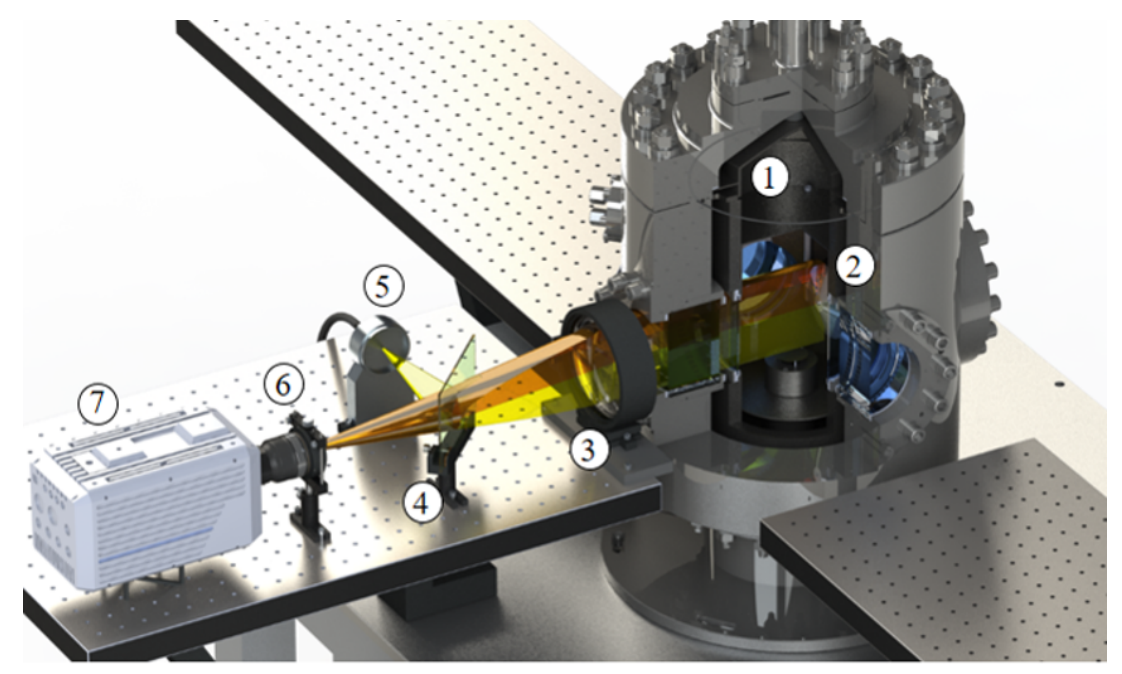
1. Test rig
5. Light font
2. Mirror
6. Diaphragm
3. Convex Lens
7. Fast camera
4. Beam splitter

Figure 2: Optical setup for double-pass Schlieren

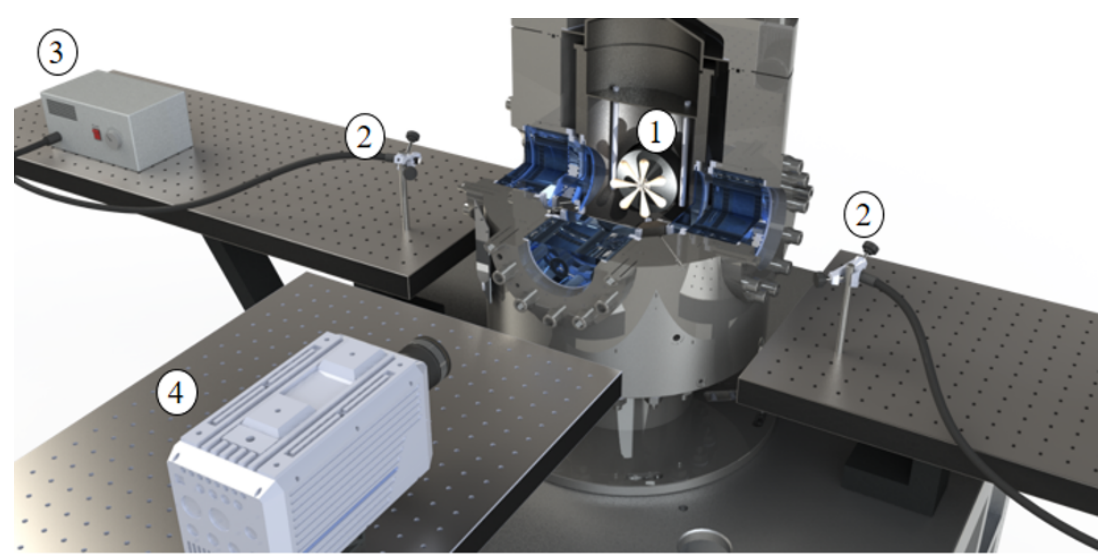

1. Test rig

3. Light font

2. Light source

4. Fast camera

Figure 3: Optical setup for MIE-Scattering 


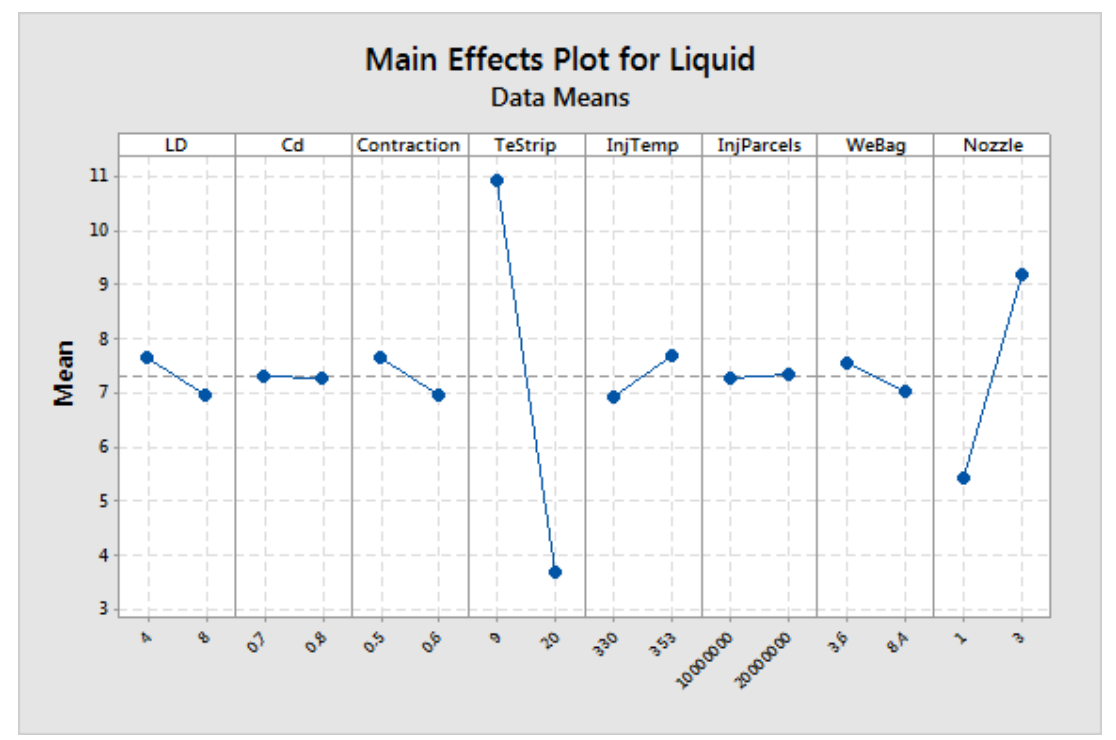

(a) Main Effects Plot for Liquid

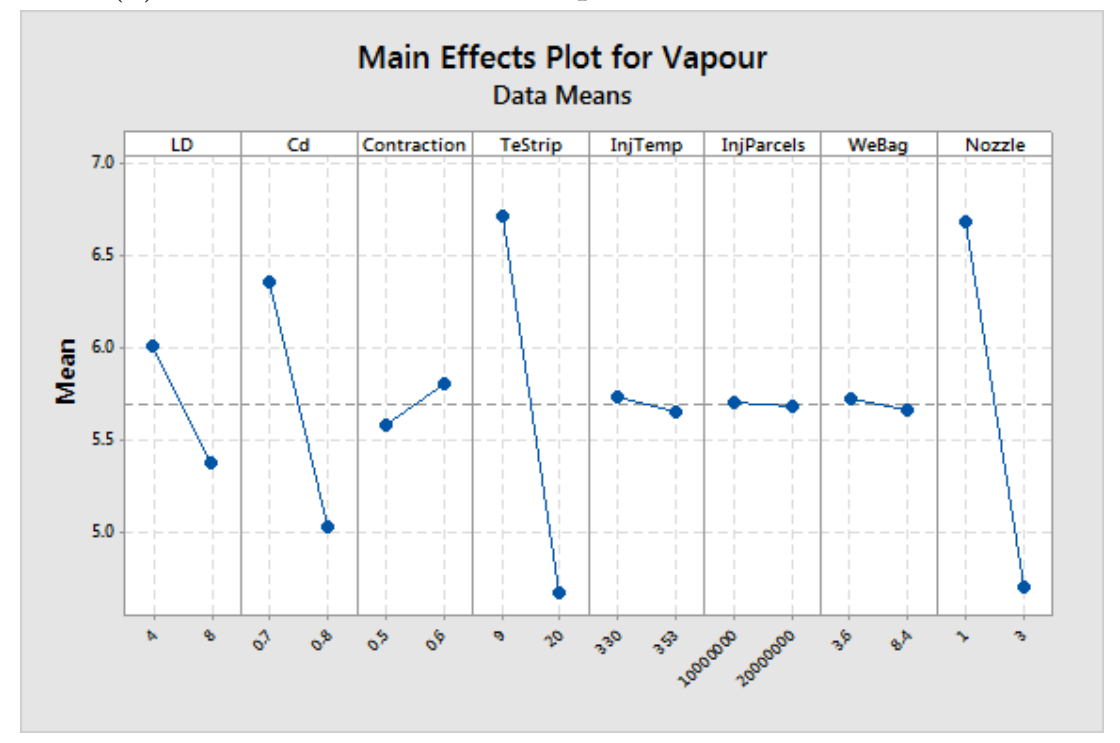

(b) Main Effects Plot for Vapor

Figure 4: Parameters effects.

Published in Applied Thermal Engineering, Volume 137, 5 June 2018, Pages 721-728 


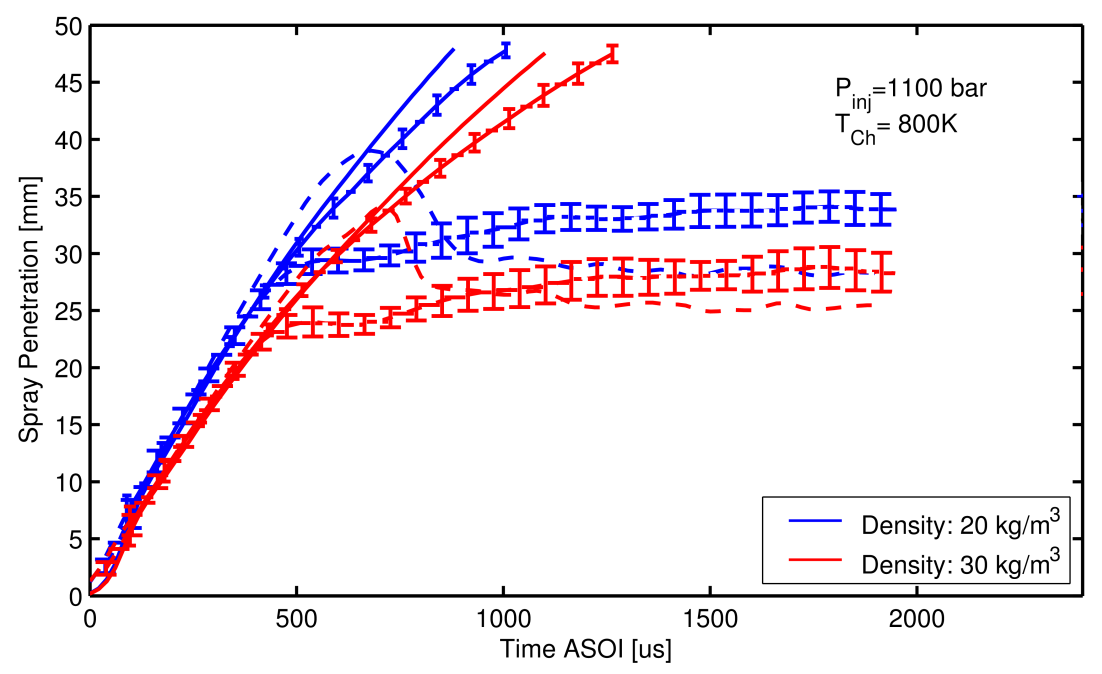

(a)

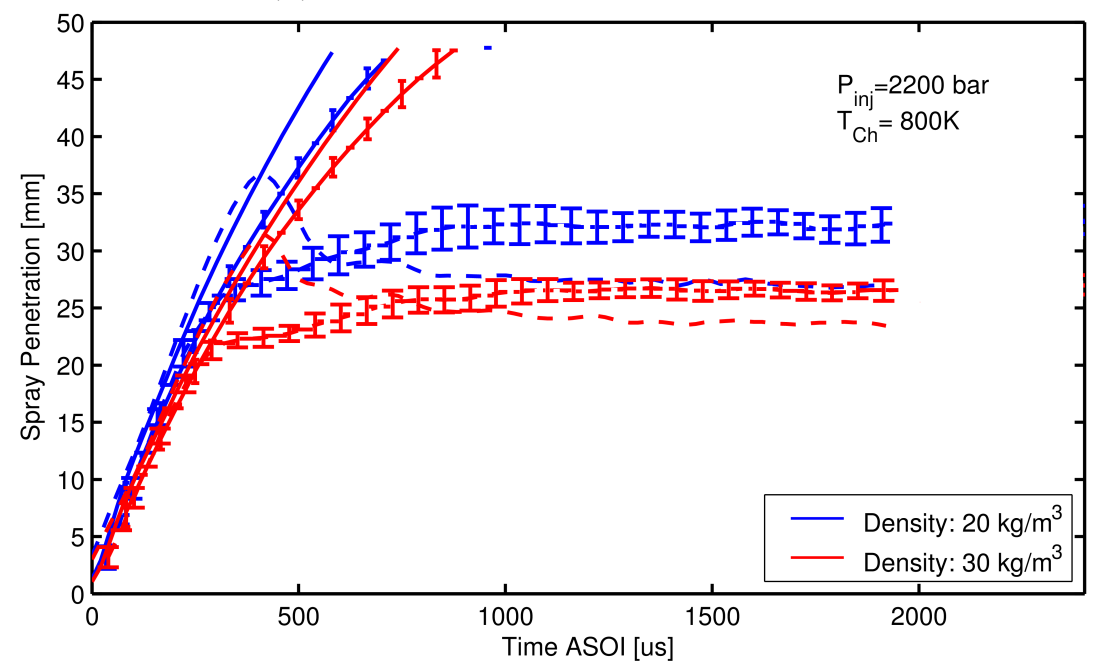

(b)

Figure 5: Influence of ambient density at 1100 (a) and 2200 bar (b). Experiments (lines with error bars), CFD (lines w/o error bars), vapor (line), liquid (Dash line) 


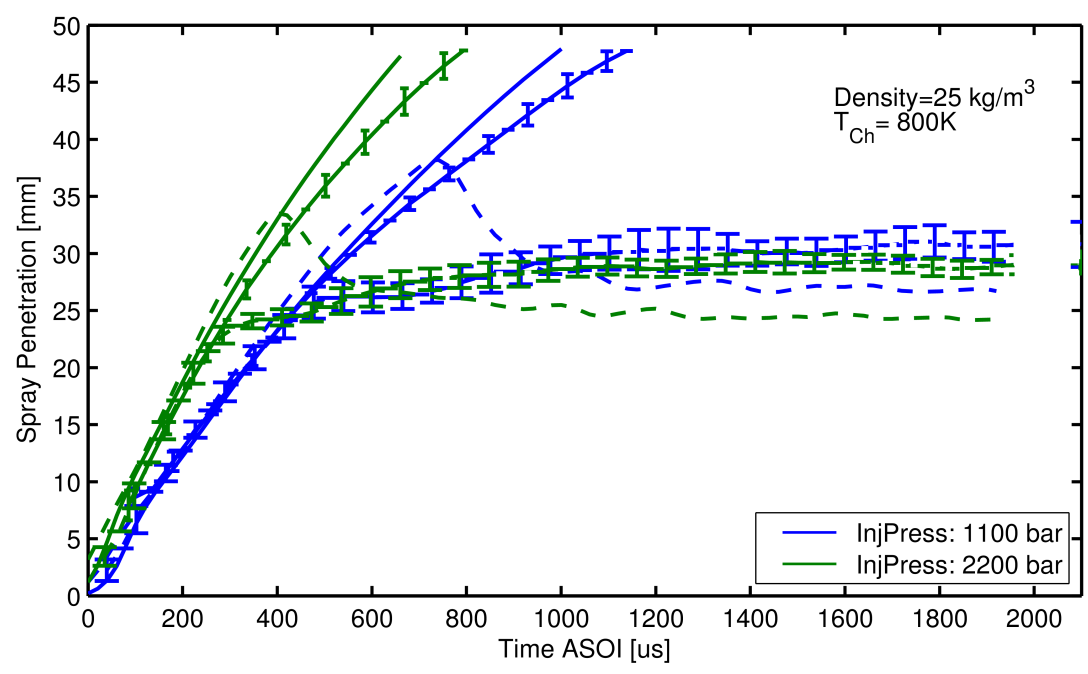

Figure 6: Influence of injection pressure. Experiments (lines with error bars), CFD (lines w/o error bars), vapor (line), liquid (Dash line)

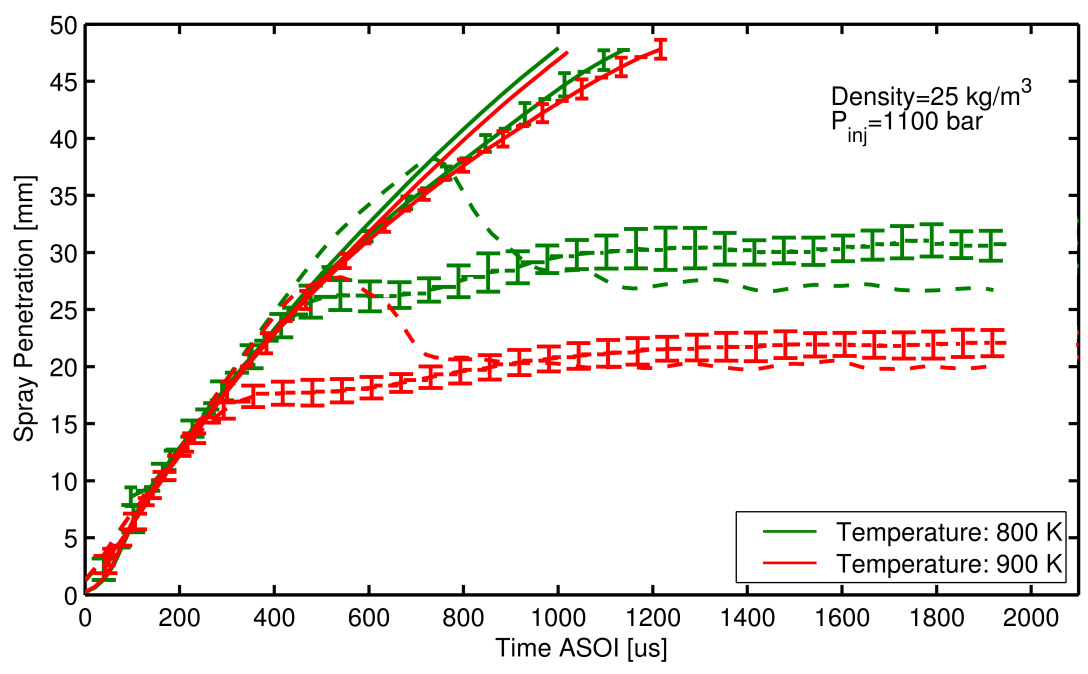

Figure 7: Effect of ambient temperature. Experiments (lines with error bars),CFD (lines w/o error bars), vapor (line), liquid (Dash line) 
Published in Applied Thermal Engineering, Volume 137, 5 June 2018, Pages 721-728

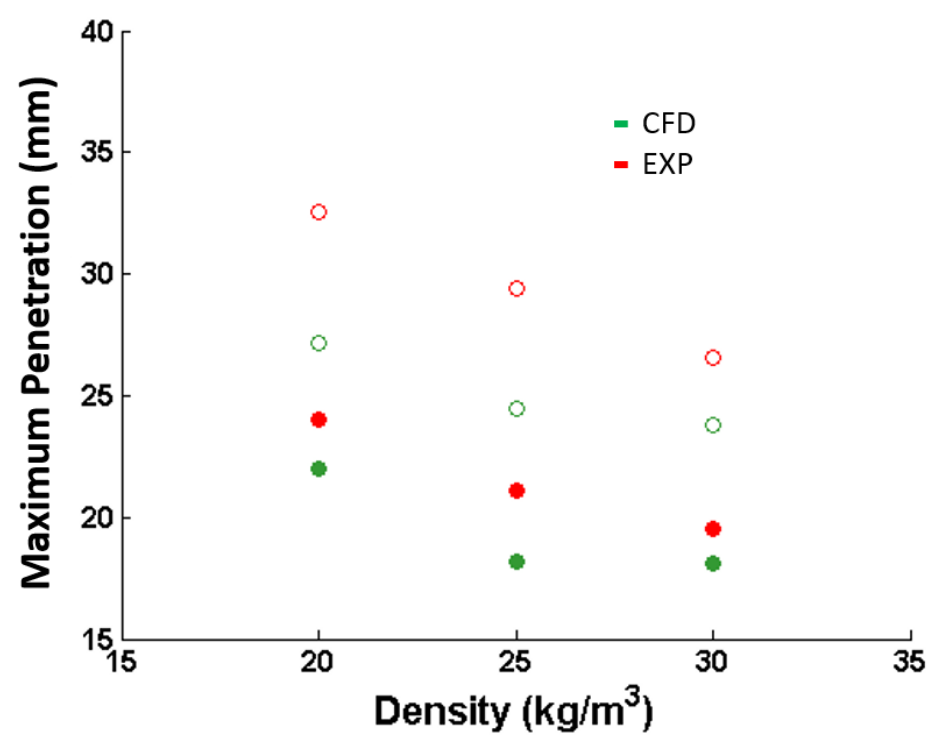

Figure 8: Effect of density and temperature on liquid length Pinj=2200bar. $\mathrm{T}=800 \mathrm{~K}$ (Markers unfilled), $\mathrm{T}=900 \mathrm{~K}$ (Markers filled)

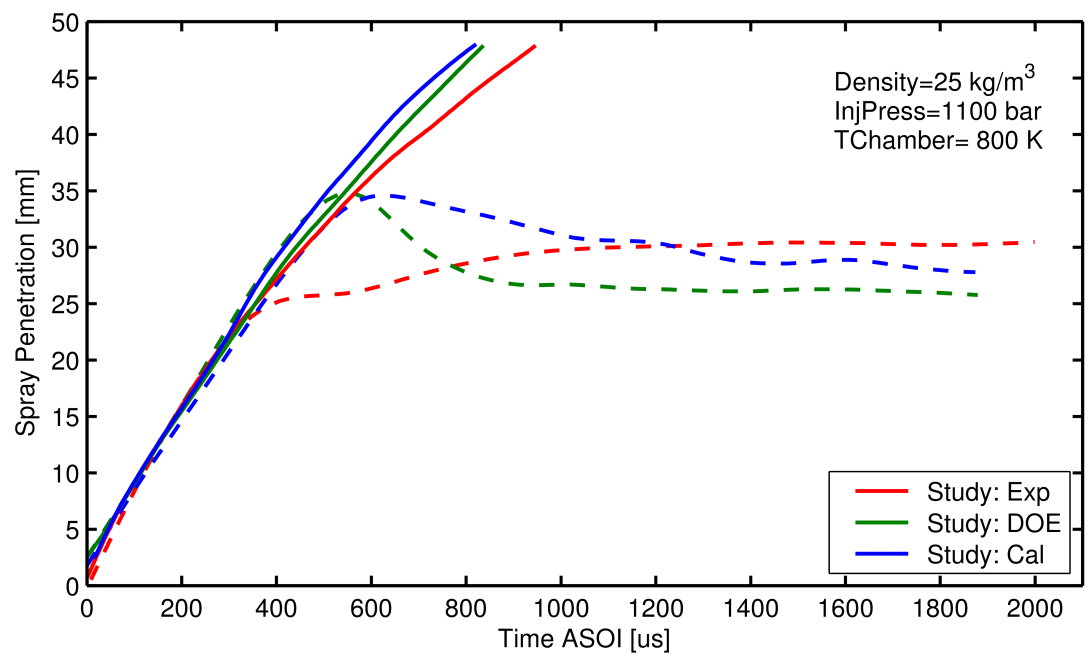

Figure 9: Calibration vs experiments. Vapor (Solid line), liquid (Dash line) 